\title{
Rigid probe solutes in a smectic- $A$ liquid crystal: An unconventional route to the latter's positional order parameters
}

\author{
Maria Enrica Di Pietro, Giorgio Celebre, and Giuseppina De Luca \\ Dipartimento di Chimica, Università della Calabria, Campus di Arcavacata, Via Pietro Bucci Cubo 12C, I-87036 Rende (Cosenza), Italy \\ Giorgio Cinacchi* \\ Departamento de Física Teórica de la Materia Condensada, Universidad Autónoma de Madrid, Campus de Cantoblanco, \\ E-28049 Madrid, Spain
}

(Received 10 August 2011; published 13 December 2011; corrected 22 December 2011)

\begin{abstract}
Biphenylene and pyrene were dissolved in the nematic and smectic- $A$ phases of the liquid crystal $4,4^{\prime}$ di- $n$-heptyl-azoxybenzene and the orientational order parameters of both solutes and solvent measured via proton and deuteron nuclear-magnetic-resonance spectroscopy. This new data set was then merged with the one previously obtained, formed by 4,4'-di-chloro-benzene and naphthalene as solutes in the same solvent, and the resulting overall data set analyzed with a statistical thermodynamic density-functional theory to provide positional-orientational distribution functions of the various solutes along with the smectic solvent's positional order parameters.
\end{abstract}

DOI: 10.1103/PhysRevE.84.061703

PACS number(s): 61.30.-v

\section{INTRODUCTION}

Smectic liquid crystals are interesting phases of matter. Their constituent particles both have an orientational order, as in the more common nematic $(N)$ phase, and a quasilong-range one-dimensional positional order. Smectics indeed consist of layers, stacked one above the other. In the simplest case, the smectic- $A\left(S_{A}\right)$ phase, particles within a layer have a liquidlike short-range order and preferentially orient along the layer normal $(\hat{\boldsymbol{n}})[1]$.

Perhaps surprisingly, there is no straightforward experimental method to quantitatively assess the extent of layering. Positional order can be readily quantified through a series of parameters $\tau_{k}$, defined as [1]

$$
\tau_{k}=\frac{1}{\delta} \int_{0}^{\delta} d z \cos \left(2 \pi k \frac{z}{\delta}\right) \rho(z),
$$

with $\rho(z)$ the distribution function of $z$, the particle displacement from the midlayer position resolved along $\hat{\boldsymbol{n}}, \delta$ the layer spacing, and $k$ a positive integer. Yet the very value borne by these parameters is experimentally hard to know for a molecular smectic liquid crystal.

Diffraction techniques would appear as the most suited to investigate positional order in smectics. Indeed, they provide accurate values of the layer spacing. For a $S_{A}$ phase, $\delta$ turns out of the order of the molecular length. Unfortunately, they cannot provide as straightforwardly also the values of the parameters $\tau_{k}$. In fact, the measured quantities, the relative scattering intensities of the first, second,..., $k$ th peak, $I_{00 k}$, do turn out proportional to $\tau_{k}^{2}$, but the proportionality constant is unknown. Thus just ratios of the type $\tau_{k^{\prime}} / \tau_{k^{\prime \prime}}$ can, in principle, be confidently obtained, though, in practice, only the ratio $\tau_{2} / \tau_{1}$ is most often achievable as usually peaks up to the second only can be detected.

\footnotetext{
*giorgio.cinacchi@uam.es
}

To proceed further and eventually arrive at values of the parameters $\tau_{k}$, a Gaussian shape for $\rho(z)$ was assumed in Ref. [2]. While there has been also an attempt to link $\tau_{1}$ with the coherence length of the smectic layers, the latter accessible in a diffraction experiment [3], the procedure of Ref. [2] was the one applied most in the past [4,5]. More recently, two methods have been proposed trying to overcome its inherent difficulties.

In the first, the unknown proportionality constant has been determined by assuming a "Haller-like"extrapolation of the temperature dependence of the measured relative intensity to the absolute zero temperature [6]. This way, values of the parameter $\tau_{1}$ for a range of smectogenic materials were obtained. In the second, small-angle neutron scattering has been used to measure the absolute intensity of the first peak, while relying upon an atomistic model for the calculation of the molecular form factor [7]. This way, values of the parameter $\tau_{1}$ for the bilayered $S_{A}$ mesogen usually labeled $8 \mathrm{CB}$ were obtained.

In recent years, the problem of determining positional order parameters in smectics was also being addressed from viewpoints other than the traditional one based on diffraction techniques. Nuclear-magnetic-resonance (NMR) spectroscopy is at the heart of these new attempts.

In one case, proton NMR was used to determine the diffusion coefficients in the $N$ and $S_{A}$ phases and then these data were related to the $\tau_{k}$ 's via a theoretical model validated by computer simulation [8].

In the other cases, rigid and relatively small molecules were dissolved in a smectogenic solvent and their Saupe ordering matrices, $\boldsymbol{S}$ [1], determined via liquid-crystal NMR (LX-NMR) spectroscopy [9]. The aim of these studies is to exploit the changes that the orientational order parameters of certain solutes may undergo upon the onset of layering to get informaton on the positional order of the smectic liquidcrystalline solutions. These studies can be further subdivided in two groups depending on how they analyze solutes' $S$ 's. This analysis has important consequences on what can be 
unveiled about the structure of the smectic liquid-crystalline solutions.

One series of these studies [10] makes use of meanfield models, developed from the classic Maier-Saupe and McMillan theories [1], and their outputs are the positional order parameters of the solutes.

In the other two studies instead [11,12], a statistical thermodynamic density-functional theory (ST-DFT), developed from the classic Onsager theory [1], is used that expresses solutes' positional-orientational distribution function in terms of the solvent's positional-orientational distribution. Importantly, this allows for the determination of the positional order parameters of the solvent too. As the solutions are sufficiently dilute, these parameters should not differ from those characterizing the pure smectic solvent. The method developed in Refs. [11,12] thus offers another way to determine the positional order parameters of a smectic liquid crystal.

The present work belongs to the above-mentioned second group. New experimental data for the Saupe ordering matrices of the solutes biphenylene (BIF) and pyrene (PYR) dissolved in the $N$ and $S_{\mathrm{A}}$ phases of the liquid crystal $4,4^{\prime}$-di- $n$ heptyl-azoxybenzene (HAB) were determined. These new experimental data were then merged with those previously determined for the solutes 1,4-di-chloro-benzene (DCB) and naphthalene (NFT) dissolved in the same solvent [12] to form a larger set, with which potentialities and limits of the ST-DFT-based methodology can be better assessed. In the next section, the latter is briefly recalled, while Sec. III contains a concise description of the new LX-NMR experiments and presentation of the data acquired from them. Section IV thoroughly discusses the ST-DFT based analysis of the overall experimental data set. Conclusions are drawn and future perspective outlined in Sec. V.

\section{RECALL OF THE METHODOLOGY}

In this section, the ST-DFT based methodology is succinctly recalled. For all details, the reader is referred to Refs. [11,12]. The basic equation coming out from the ST-DFT is

$$
\begin{aligned}
\ln \rho_{\sigma}(z, \Omega)= & -2 \Psi \int_{-\infty}^{+\infty} d z^{\prime} d \Omega^{\prime} \rho_{\Sigma}\left(z^{\prime}, \Omega^{\prime}\right) \\
& \times A_{\sigma \Sigma}\left(z-z^{\prime}, \Omega, \Omega^{\prime}\right)-\ln \Xi .
\end{aligned}
$$

The meaning of the terms entering this equation is explained here below.

(i) $\rho_{\sigma}(z, \Omega)$ is the probability density to find a solute molecule $\sigma$ in the state defined by the position of its center of mass along $\hat{\boldsymbol{n}}, z$, and its orientation, specified by the usual set of Euler angles [13] collected under the symbol $\Omega$.

(ii) In close analogy with what is written above, $\rho_{\Sigma}\left(z^{\prime}, \Omega^{\prime}\right)$ is the probability density to find a solvent molecule $\Sigma$ in the positional-orientational state defined by $z^{\prime}$ and $\Omega^{\prime}$.

(iii) The quantity $A_{\sigma \Sigma}\left(z-z^{\prime}, \Omega, \Omega^{\prime}\right)$ measures solutesolvent interactions. More specifically, it is given by minus the integral of the Mayer function $M_{\sigma \Sigma}\left(\boldsymbol{R}, z-z^{\prime}, \Omega, \Omega^{\prime}\right)$ over $\boldsymbol{R}$, the vector of the distance between the two molecules' centers of mass resolved perpendicularly to $\hat{\boldsymbol{n}} ; M_{\sigma \Sigma}(\boldsymbol{R}, z-$ $\left.z^{\prime}, \Omega, \Omega^{\prime}\right)=\exp \left[-\frac{u_{\sigma \Sigma}\left(\boldsymbol{R}, z-z^{\prime}, \Omega, \Omega^{\prime}\right)}{k_{B} T}\right]-1$, with $k_{B}$ the Boltzmann constant, $T$ the temperature, and $u_{\sigma \Sigma}\left(\boldsymbol{R}, z-z^{\prime}, \Omega, \Omega^{\prime}\right)$ the intermolecular solute-solvent interaction potential energy function [14].

(iv) The factor $\Psi$ helps correct in an effective way for the neglect of higher-order (virial) terms involving ever more complicated integrals.

(v) $\Xi$ is the normalization constant, ensuring that $\int d z d \Omega \rho_{\sigma}(z, \Omega)=1$.

Equation (2) is valid for very dilute solutions. Under these conditions, the positional-orientational distribution function of a solute is linked to the positional-orientational distribution function of the solvent via the function $A_{\sigma \Sigma}$.

Once an approximation for solute-solvent interactions has been set out and $\rho_{\Sigma}(z, \Omega)$ suitably parametrized, these parameters can be determined by fitting the experimental Saupe ordering matrices, obtained by means of LX-NMR, to those calculated by the ST-DFT.

If $\left[S_{\mathrm{ab}}\right]_{\sigma}^{\text {expt }}$ is the $a b$ element of the experimental Saupe ordering matrix of the solute $\sigma$, the corresponding calculated quantity, $\left[S_{\mathrm{ab}}\right]_{\sigma}^{\mathrm{calc}}$, is

$\left[S_{\mathrm{ab}}\right]_{\sigma}^{\mathrm{calc}}=\int d z d \Omega \rho_{\sigma}(z, \Omega)\left[\frac{3}{2}(\hat{\boldsymbol{a}} \cdot \hat{\boldsymbol{n}})(\hat{\boldsymbol{b}} \cdot \hat{\boldsymbol{n}})-\frac{\delta_{\mathrm{ab}}}{2}\right]$,

with $\hat{\boldsymbol{a}}$ the $a$ th axis of the molecular reference frame and $\delta_{\mathrm{ab}}$ the Krönecker symbol. What one is required to minimize is then a sum of the following kind:

$$
\tilde{\chi}^{2}=\frac{1}{v} \sum_{\sigma} \sum_{\mathrm{ab}}\left\{\left[S_{\mathrm{ab}}\right]_{\sigma}^{\mathrm{expt}}-\left[S_{\mathrm{ab}}\right]_{\sigma}^{\mathrm{calc}}\right\}^{2}
$$

with $v$ the number of data minus the number of parameters.

\section{EXPERIMENTS}

Two dilute solutions, approximately $3 \%$ by mole fraction, were prepared dissolving the rigid solutes $\mathrm{BIF}$ and PYR in a mixture of $\mathrm{HAB}$ and $\mathrm{HAB}-\mathrm{d}_{4}$, the latter being the version deuterated in the positions ortho with respect to the two alkyl chains. The solutes BIF and PYR and the solvent HAB were purchased from Aldrich, while the deuterated sample of $\mathrm{HAB}-\mathrm{d}_{4}$ was provided by C. A. Veracini (Dipartimento di Chimica, Università di Pisa). In Fig. 1, the structures of the solvent and all solute molecules considered in this work are reported together with the molecular reference frame chosen for these solute molecules.
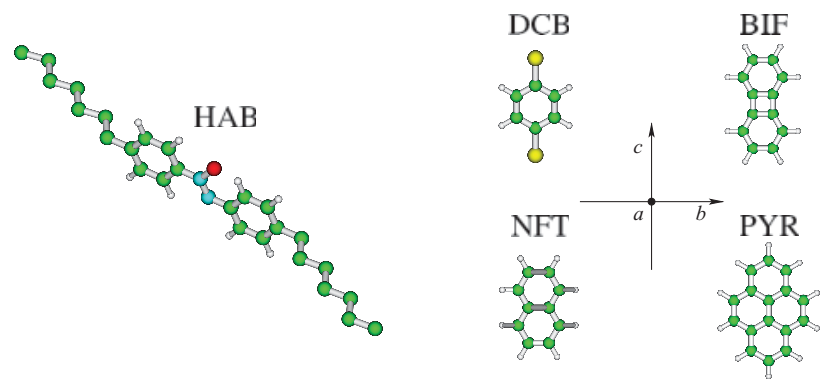

FIG. 1. (Color online) "Ball-and-stick" structures of the solvent and solute molecules, referred to with their respective label defined in the text. Shown are also the axes, $a, b$ and $c$, of the molecular reference frame adopted for the solute molecules. 


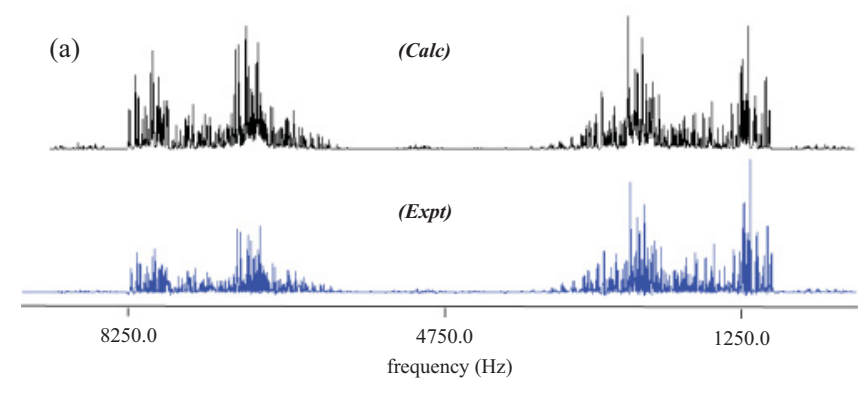

(b)
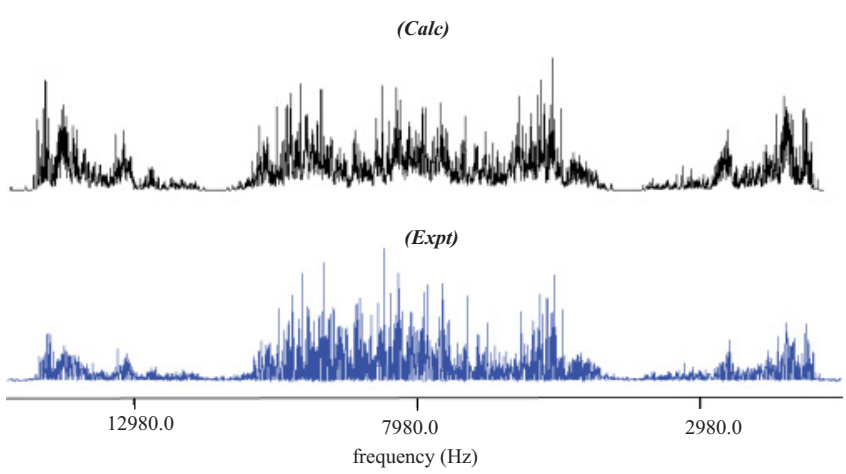

FIG. 2. (Color online) Calculated and experimental ${ }^{1} \mathrm{H}-\mathrm{NMR}$ spectra of (a) BIF and (b) PYR in HAB at $T^{*}=0.93$.
On both samples, ${ }^{1} \mathrm{H}$ spectra of the solutes and ${ }^{2} \mathrm{H}$ spectra of the solvent were recorded at different common reduced temperatures $T^{*}=T / T_{N I}$, with $T_{N I}$ the nematic-isotropic phase-transition temperature. The temperature range spanned both $N$ and $S_{A}$ liquid-crystal phases. All anisotropic spectra were recorded on a Bruker Avance $500 \mathrm{MHz}$ spectrometer working at a field strength of $11.74 \mathrm{~T}$ and equipped with a temperature control unit. All proton spectra were analyzed using the iterative computer program ARCANA [15]. Figure 2 reports, as an example, the calculated and the experimental ${ }^{1} \mathrm{H}$ spectra in $\mathrm{HAB}$ at $T^{*}=0.93$ for BIF and PYR, respectively.

The final experimental data, that is the hydrogen atom $i$-hydrogen atom $j$ residual dipolar couplings, $D_{i j}$ 's, are given, for each temperature, in Table I for BIF and in Table II for PYR. Tables III and IV report the $S$ matrices obtained from the residual dipolar coupling data for the two solutes. Following the procedure already described in Ref. [12], the deuterium spectra of the solvent were used to obtain the numerical values of the solvent's second-rank orientational order parameter $\eta$. These are listed in Table V. For the sake of completeness, we need to mention an interesting approach, recently proposed, to determine the orientational order of the pure HAB [16]. This involves the simultaneous analysis of several NMR observables $\left({ }^{13} \mathrm{C}\right.$ chemical shift anisotropies, ${ }^{1} \mathrm{H}-{ }^{2} \mathrm{H}$ and ${ }^{13} \mathrm{C}-{ }^{2} \mathrm{H}$ dipolar couplings, and ${ }^{2} \mathrm{H}$ quadrupolar splittings) supplemented by quantum chemical density-functional theory calculations, in order to derive HAB orientational order parameters supposedly more reliable than

TABLE I. Experimental residual dipolar couplings (Hz) of BIF in HAB at different reduced temperatures. The transition between the nematic and smectic- $A$ phases is recorded at about $T^{*}=0.94$. The chemical structure provides the hydrogen atom numbering.

\begin{tabular}{|c|c|c|c|c|c|c|c|}
\hline$T^{*}$ & $D_{12}$ & $D_{13}$ & $D_{14}$ & ${ }^{5}$ & $D_{16}$ & $D_{17}$ & $D_{18}$ \\
\hline 0.97 & $-1349.93 \pm 0.08$ & $-75.07 \pm 0.11$ & $13.24 \pm 0.31$ & $-39.94 \pm 0.33$ & $-60.57 \pm 0.10$ & $-125.54 \pm 0.09$ & $-529.65 \pm 0.25$ \\
\hline 0.96 & $-1444.93 \pm 0.08$ & $-78.38 \pm 0.12$ & $17.11 \pm 0.36$ & $-40.76 \pm 0.35$ & $-65.06 \pm 0.12$ & $-135.06 \pm 0.10$ & $-568.15 \pm 0.25$ \\
\hline 0.95 & $-1512.69 \pm 0.06$ & $-80.26 \pm 0.08$ & $19.47 \pm 0.24$ & $-43.02 \pm 0.24$ & $-68.11 \pm 0.08$ & $-141.27 \pm 0.07$ & $-595.34 \pm 0.17$ \\
\hline 0.94 & $-1572.97 \pm 0.05$ & $-83.03 \pm 0.06$ & $19.83 \pm 0.19$ & $-45.46 \pm 0.22$ & $-70.62 \pm 0.06$ & $-147.15 \pm 0.05$ & $-620.20 \pm 0.14$ \\
\hline 0.93 & $-1634.03 \pm 0.04$ & $-88.10 \pm 0.05$ & $20.25 \pm 0.15$ & $-46.78 \pm 0.15$ & $-73.46 \pm 0.05$ & $-152.51 \pm 0.05$ & $-642.55 \pm 0.11$ \\
\hline 0.92 & $-1661.51 \pm 0.04$ & $-90.88 \pm 0.04$ & $18.68 \pm 0.16$ & $-48.01 \pm 0.13$ & $-74.73 \pm 0.04$ & $-154.93 \pm 0.04$ & $-652.48 \pm 0.10$ \\
\hline 0.91 & $-1679.02 \pm 0.04$ & $-93.08 \pm 0.05$ & $17.46 \pm 0.16$ & $-48.67 \pm 0.17$ & $-75.38 \pm 0.05$ & $-156.39 \pm 0.04$ & $-658.69 \pm 0.11$ \\
\hline$T^{*}$ & $D_{23}$ & $D_{26}$ & $D_{27}$ & & & & \\
\hline 0.97 & $124.17 \pm 0.31$ & $-44.91 \pm 0.31$ & $-56.90 \pm 0.25$ & & & & \\
\hline 0.96 & $151.58 \pm 0.34$ & $-49.29 \pm 0.33$ & $-60.69 \pm 0.26$ & & & & \\
\hline 0.95 & $171.23 \pm 0.25$ & $-51.48 \pm 0.24$ & $-63.94 \pm 0.18$ & & & & \\
\hline 0.94 & $183.44 \pm 0.18$ & $-52.78 \pm 0.20$ & $-66.58 \pm 0.16$ & & & & \\
\hline 0.93 & $175.21 \pm 0.15$ & $-55.44 \pm 0.15$ & $-69.26 \pm 0.11$ & & & & \\
\hline 0.92 & $167.01 \pm 0.13$ & $-56.02 \pm 0.13$ & $-70.24 \pm 0.10$ & & & & \\
\hline 0.91 & $158.09 \pm 0.15$ & $-56.78 \pm 0.15$ & $-70.53 \pm 0.11$ & & & & \\
\hline
\end{tabular}


TABLE II. Experimental residual dipolar couplings (Hz) of PYR in HAB at different reduced temperatures. The transition between nematic and smectic- $A$ phase is recorded at about $T^{*}=0.94$. The chemical structure provides the hydrogen atom numbering.

\begin{tabular}{|c|c|c|c|c|c|c|c|}
\hline$T^{*}$ & $D_{12}$ & $D_{13}$ & 8 & 1 & 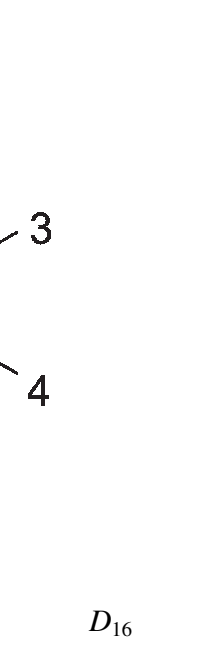 & & \\
\hline $\begin{array}{l}0.97 \\
0.96 \\
0.95 \\
0.94 \\
0.93 \\
0.92 \\
0.91\end{array}$ & $\begin{array}{l}-56.82 \pm 0.10 \\
-60.24 \pm 0.09 \\
-60.71 \pm 0.08 \\
-64.21 \pm 0.07 \\
-65.42 \pm 0.07 \\
-68.87 \pm 0.07 \\
-69.39 \pm 0.08\end{array}$ & $\begin{array}{l}-84.65 \pm 0.14 \\
-86.89 \pm 0.12 \\
-91.04 \pm 0.12 \\
-94.70 \pm 0.09 \\
-96.13 \pm 0.09 \\
-99.92 \pm 0.11 \\
-102.03 \pm 0.11\end{array}$ & $\begin{array}{l}-154.98 \pm 0.14 \\
-161.67 \pm 0.12 \\
-166.66 \pm 0.11 \\
-171.01 \pm 0.10 \\
-175.54 \pm 0.09 \\
-178.06 \pm 0.11 \\
-183.01 \pm 0.12\end{array}$ & $\begin{array}{l}-554.17 \pm 0.09 \\
-569.45 \pm 0.08 \\
-578.34 \pm 0.07 \\
-586.90 \pm 0.06 \\
-592.99 \pm 0.05 \\
-597.84 \pm 0.07 \\
-601.79 \pm 0.07\end{array}$ & $\begin{array}{l}-43.02 \pm 0.16 \\
-46.20 \pm 0.15 \\
-46.87 \pm 0.13 \\
-49.80 \pm 0.10 \\
-51.36 \pm 0.09 \\
-53.65 \pm 0.11 \\
-54.57 \pm 0.11\end{array}$ & & \\
\hline$T^{*}$ & $D_{23}$ & $D_{24}$ & $D_{25}$ & $D_{27}$ & $D_{28}$ & $D_{29}$ & $D_{210}$ \\
\hline $\begin{array}{l}0.97 \\
0.96 \\
0.95 \\
0.94 \\
0.93 \\
0.92 \\
0.91\end{array}$ & $\begin{array}{l}-1654.15 \pm 0.09 \\
-1733.40 \pm 0.07 \\
-1798.50 \pm 0.06 \\
-1864.45 \pm 0.06 \\
-1911.60 \pm 0.05 \\
-1974.15 \pm 0.06 \\
-2015.29 \pm 0.06\end{array}$ & $\begin{array}{l}-554.17 \pm 0.09 \\
-569.45 \pm 0.08 \\
-578.34 \pm 0.07 \\
-586.90 \pm 0.06 \\
-592.99 \pm 0.05 \\
-352.22 \pm 0.07 \\
-359.85 \pm 0.07\end{array}$ & $\begin{array}{l}-115.05 \pm 0.12 \\
-118.41 \pm 0.10 \\
-123.35 \pm 0.09 \\
-127.18 \pm 0.08 \\
-131.37 \pm 0.07 \\
-135.20 \pm 0.08 \\
-139.14 \pm 0.08\end{array}$ & $\begin{array}{l}-48.67 \pm 0.16 \\
-51.07 \pm 0.14 \\
-53.88 \pm 0.12 \\
-54.04 \pm 0.10 \\
-54.44 \pm 0.10 \\
-55.30 \pm 0.12 \\
-58.66 \pm 0.13\end{array}$ & $\begin{array}{l}-37.78 \pm 0.14 \\
-39.46 \pm 0.11 \\
-39.58 \pm 0.10 \\
-42.46 \pm 0.09 \\
-43.42 \pm 0.09 \\
-43.58 \pm 0.11 \\
-44.41 \pm 0.10\end{array}$ & $\begin{array}{l}-24.12 \pm 0.15 \\
-22.40 \pm 0.11 \\
-22.31 \pm 0.10 \\
-22.20 \pm 0.09 \\
-22.02 \pm 0.08 \\
-21.12 \pm 0.10 \\
-20.44 \pm 0.10\end{array}$ & $\begin{array}{c}-4.46 \pm 0.16 \\
0.27 \pm 0.14 \\
1.70 \pm 0.12 \\
4.88 \pm 0.11 \\
10.58 \pm 0.10 \\
12.30 \pm 0.12 \\
15.41 \pm 0.12\end{array}$ \\
\hline$T^{*}$ & $D_{34}$ & $D_{38}$ & $D_{39}$ & & & & \\
\hline $\begin{array}{l}0.97 \\
0.96 \\
0.95 \\
0.94 \\
0.93 \\
0.92 \\
0.91\end{array}$ & $\begin{array}{l}-2269.34 \pm 0.12 \\
-2383.02 \pm 0.09 \\
-2474.10 \pm 0.08 \\
-2572.08 \pm 0.07 \\
-2642.06 \pm 0.07 \\
-2734.75 \pm 0.08 \\
-2796.91 \pm 0.09\end{array}$ & $\begin{array}{l}-12.84 \pm 0.19 \\
-12.40 \pm 0.16 \\
-12.06 \pm 0.14 \\
-11.05 \pm 0.12 \\
-10.79 \pm 0.11 \\
-10.49 \pm 0.15 \\
-10.35 \pm 0.15\end{array}$ & $\begin{array}{c}-1.23 \pm 0.20 \\
-0.82 \pm 0.17 \\
0.37 \pm 0.13 \\
0.76 \pm 0.12 \\
1.88 \pm 0.11 \\
4.03 \pm 0.16 \\
4.25 \pm 0.15\end{array}$ & & & & \\
\hline
\end{tabular}

the ones obtained by the traditional approach, based on the simpler analysis of ${ }^{2} \mathrm{H}$ spectra. That more elaborated approach could not be applied in the present case, though, as solutions of $\mathrm{HAB}$, and not the pure $\mathrm{HAB}$, are dealt with here: this implies that, in principle, the aromatic signals of ${ }^{13} \mathrm{C} N \mathrm{NMR}$ spectra of the solutes may interfere with those of HAB. Therefore, it was decided to pursue with the traditional approach. It is anyway nice to see that the data of Table $\mathrm{V}$ are consistent with those of Fig. 6 of Ref. [16].

\section{DATA ANALYSIS AND DISCUSSION}

The analysis of the whole set of experimental data, composed of the $S$ 's of DCB, NFT, BIF, and PYR as a function of temperature, started with a choice of a model for solute-solvent interactions. In analogy with what was done in the previous work [12], both solute and solvent molecules were described at the atomistic, chemically detailed level (Fig. 1). Yet atom-atom interactions were taken of the hard sphere type, with each sphere radius equal to the corresponding atom (or group: the united atom approximation was used for methylene and methyl groups in the alkyl chains of HAB) van der Waals radius [17]. The model chosen was thought of as a compromise between: on one hand, the desire of preserving the individuality of each molecule together with the knowledge that fluid structure is primarily determined by short-range interactions (e.g. Ref. [18]) and, among these, the ones of the shape-and-size type are usually dominating; on 
TABLE III. Elements of the Saupe ordering matrix for BIF in $\mathrm{HAB}$ as a function of reduced temperature.

\begin{tabular}{lcc}
\hline \hline$T^{*}$ & $S_{c c}$ & $S_{b b}-S_{a a}$ \\
\hline 0.97 & $0.2560 \pm 0.0003$ & $0.2261 \pm 0.0004$ \\
0.96 & $0.2767 \pm 0.0003$ & $0.2382 \pm 0.0002$ \\
0.95 & $0.2882 \pm 0.0003$ & $0.2469 \pm 0.0003$ \\
0.94 & $0.3002 \pm 0.0003$ & $0.2561 \pm 0.0004$ \\
0.93 & $0.3111 \pm 0.0003$ & $0.2689 \pm 0.0004$ \\
0.92 & $0.3158 \pm 0.0003$ & $0.2757 \pm 0.0003$ \\
0.91 & $0.3210 \pm 0.0003$ & $0.2855 \pm 0.0003$ \\
\hline \hline
\end{tabular}

the other hand, the need to keep calculations as simple as possible. For the same reason, the solvent was allowed to adopt a single conformation, the one shown in Fig. 1, having the central core planar, the dihedral angles defined by the first two methylene groups of the chains and the corresponding phenyl ring set, respectively, at $\pm 90^{\circ}$ and the two chains in an all trans conformation.

The second step was the parametrization of the solvent distribution function. In analogy with what was done in the previous work, $\rho_{\Sigma}\left(z^{\prime}, \Omega^{\prime}\right)$ was written as the product of positional and orientational terms:

$$
\rho_{\Sigma}\left(z^{\prime}, \Omega^{\prime}\right)=\frac{\exp \left[\lambda \cos \left(2 \pi \frac{z^{\prime}}{\delta}\right)\right]}{\Upsilon_{\lambda}} \frac{\exp \left[\gamma P_{2}\left(\cos \theta^{\prime}\right)\right]}{\Upsilon_{\gamma}} .
$$

In the equation above, $\lambda$ is the parameter regulating the steepness of the positional distribution function, while $\gamma$ is that one regulating the steepness of the orientational distribution function and bearing a one-to-one correspondence with the solvent's orientational order parameter $\eta ; \theta^{\prime}$ is the angle formed by a solvent molecule's main axis with $\hat{\boldsymbol{n}} ; \Upsilon_{\lambda}$ and $\Upsilon_{\gamma}$ are the two respective normalization constants. Since $\eta$ is known from the ${ }^{2} \mathrm{H}-\mathrm{NMR}$ measurements, the solvent's orientational distribution function is completely determined under this approximation. It can thus be used to orientationally average the above-mentioned function $A_{\sigma \Sigma}\left(z-z^{\prime}, \Omega, \Omega^{\prime}\right)$ leading to the function $\alpha(z, \Omega \mid \eta)$, parametrically depending on the solvent's orientational order parameter.

To this point, solute's distribution functions depend, in general, on three parameters: $\Psi, \lambda$, and $\delta$. These are determined by fitting the predicted elements of solute Saupe ordering matrices with those obtained experimentally.

Two series of fittings were carried out. In the first, the phase was assumed $N$ throughout the temperature range explored.

TABLE IV. Elements of the Saupe ordering matrix for PYR in $\mathrm{HAB}$ as a function of reduced temperature.

\begin{tabular}{lcc}
\hline \hline$T^{*}$ & $S_{c c}$ & $S_{b b}-S_{a a}$ \\
\hline 0.97 & $0.2876 \pm 0.0016$ & $0.2903 \pm 0.0028$ \\
0.96 & $0.3038 \pm 0.0027$ & $0.2998 \pm 0.0023$ \\
0.95 & $0.3150 \pm 0.0029$ & $0.3080 \pm 0.0023$ \\
0.94 & $0.3271 \pm 0.0028$ & $0.3152 \pm 0.0022$ \\
0.93 & $0.3375 \pm 0.0025$ & $0.3196 \pm 0.0019$ \\
0.92 & $0.3477 \pm 0.0028$ & $0.3262 \pm 0.0024$ \\
0.91 & $0.3561 \pm 0.0027$ & $0.3300 \pm 0.0020$ \\
\hline \hline
\end{tabular}

TABLE V. Temperature dependence of $\eta$, the solvent's orientational order parameter.

\begin{tabular}{lc}
\hline \hline$T^{*}$ & $\eta$ \\
\hline 0.97 & 0.65 \\
0.96 & 0.68 \\
0.95 & 0.71 \\
0.94 & 0.72 \\
0.93 & 0.75 \\
0.92 & 0.78 \\
0.91 & 0.80 \\
\hline \hline
\end{tabular}

In this case, $\lambda$ was set equal to 0 and $\delta$ thus left undefined, so that $\Psi$ was the sole parameter left to vary. In the second, the phase was assumed $S_{A}$ throughout the temperature range explored. In this case, $\lambda$ was varied together with $\Psi$, while $\delta$ was fixed at the value of $28.9 \AA$ as reported in a past diffraction experiment [19]. As the experimental data set was large enough, there was no need to make any extrapolation of the result for $\Psi$ in the $N$ phase down to the $S_{A}$ phase, as done in the previous work [12]. There, the values of $\Psi$ obtained in the $N$ phase were linearly extrapolated into the $S_{A}$ phase and only the parameter $\lambda$ was varied in the layered phase. The few experimental data available at that time compelled the assumption of an extrapolation, which was made linear for the sake of simplicity.

Table VI gives the values of the parameters resulting from the two series of fittings. It can be noticed how the trend of the $\tilde{\chi}^{2}$ 's is in good agreement with the known phase sequence. While $\tilde{\chi}^{2}{ }_{N}$ increases with decreasing temperature, $\tilde{\chi}^{2}{ }_{S_{A}}$ has in general the opposite behavior. This means that, as expected, the addition of the parameter $\lambda$ leads to progressively better fittings. Nonetheless, $\tilde{\chi^{2}}{ }_{N}$ remains smaller for the first two highest temperatures, while at $T^{*}=0.95$ the two values of $\tilde{\chi}^{2}$ are essentially equivalent. This means that, for the three highest temperatures, the extra parameter added does not improve the quality of the fitting obtained by varying the sole parameter $\Psi$. This is consistent with the $N$ character of the liquid-crystalline solutions for $T^{*} \geqslant 0.95$. The addition of $\lambda$ leads to fittings of a substantially better quality for $T^{*} \leqslant 0.94$, where the liquidcrystalline solutions are indeed in the $S_{\mathrm{A}}$ phase. While $\tilde{\chi}^{2} S_{A}$ does decrease in general with temperature, it does not do so at the lowest temperature considered, at which its value is comparable to the one obtained at $T^{*}=0.95$. There is no evident reason for that at the moment.

TABLE VI. Parameters of the two series of fitting as a function of reduced temperature.

\begin{tabular}{lccccc}
\hline \hline$T^{*}$ & $10^{4} \tilde{\chi}^{2}{ }_{N}$ & $10^{4} \Psi_{N}$ & $10^{4} \tilde{\chi}^{2} S_{A}$ & $10^{4} \Psi_{S_{A}}$ & $\lambda$ \\
\hline 0.97 & 5.73 & 3.62 & 6.66 & 3.82 & 0.35 \\
0.96 & 5.90 & 3.15 & 6.52 & 3.74 & 0.67 \\
0.95 & 6.11 & 2.71 & 5.93 & 3.67 & 0.94 \\
0.94 & 6.60 & 2.12 & 4.92 & 3.34 & 1.27 \\
0.93 & 7.94 & 1.66 & 3.77 & 3.13 & 1.66 \\
0.92 & 9.65 & 1.39 & 3.56 & 2.93 & 1.95 \\
0.91 & 12.11 & 1.13 & 5.88 & 2.52 & 2.24 \\
\hline \hline
\end{tabular}




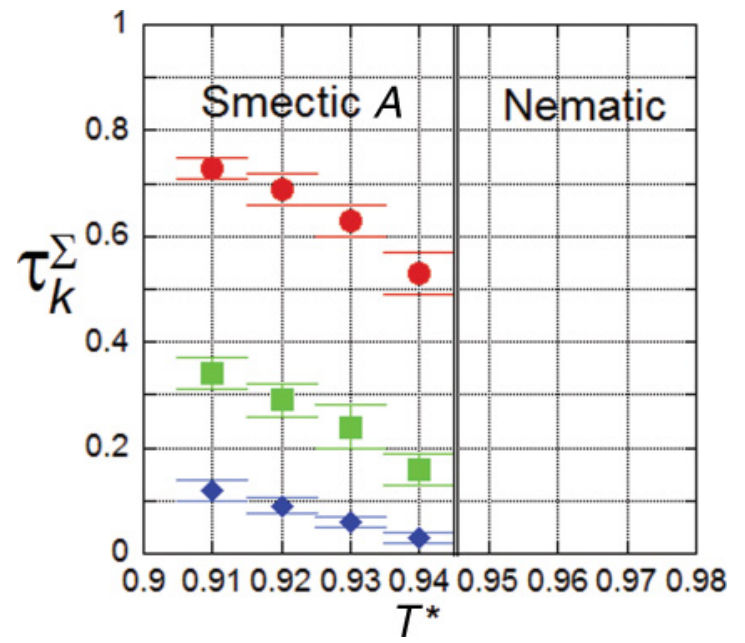

FIG. 3. (Color online) Solvent's positional order parameters $\tau_{k}$ as a function of reduced temperature: $k=1$ (red circles), $k=2$ (green squares), and $k=3$ (blue diamonds).

The main result of the analysis of the experimental $S$ 's are the solvent's $\tau_{k}$ 's. Figure 3 shows them for $k=1,2,3$ as a function of temperature. The error in these parameters was estimated assuming a $10 \%$ error in the value of $\eta$. Uncertainty in this quantity was indeed observed to be the main contribution to the uncertainty affecting the final results.

By-products of the analysis are the solutes' positional-orientational distribution functions Eq. (2). From these functions, positional order parameters of the solutes are calculated. Figure 4 shows them. Noteworthy is the negative value of $\tau_{1}$, indicating that the solutes prefer to reside in the interlayer regions. This confirms what was previously found elsewhere [10-12]. The positive value of $\tau_{2}$ is just a further reflection of the layered nature of the $S_{A}$ phase.

\section{CONCLUSIONS}

This work exploits the LX-NMR experimental technique coupled with a statistical thermodynamic density-functional theory to get values of the positional order parameters of a typical molecular smectic- $A$ liquid crystal. These parameters

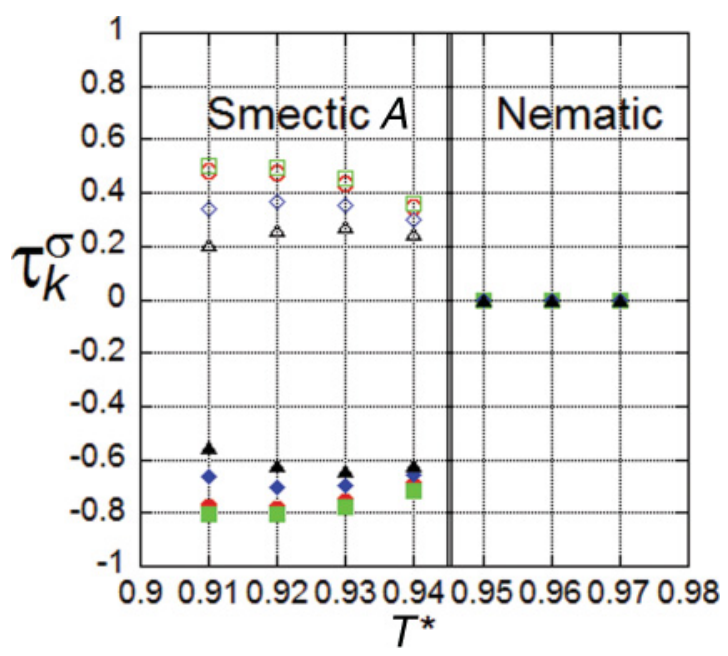

FIG. 4. (Color online) Solutes' positional order parameters as a function of reduced temperature: DCB (red circles), NFT (green squares), BIF (blue diamonds), and PYR (black triangles). Full symbols correspond to $k=1$, while empty symbols to $k=2$.

are the key quantities characterizing this type of layered mesophase. To devise a procedure to obtain them is a goal of basic importance for all liquid-crystal science. The methodology used in this work is intimately different from the ones used in past and recent years, all relying on diffraction experiments. Thus, it offers a complementary tool to achieve the above-mentioned goal. It would be interesting to compare the outcomes of the present methodology with those of any diffraction-based one. This piece of research would be a significant step forward for all liquid-crystal science.

\section{ACKNOWLEDGMENTS}

M.E.D.P. is grateful to the European Commission, the European Social Fund, and the Regione Calabria for cofunding her Ph.D. scholarship. M.E.D.P., G.C., and G.D.L. thank the University of Calabria and MIUR PRIN 2009 for financial support. G.C. acknowledges the financial support of the Spanish Ministry of Research via a Ramón y Cajal research fellowship.
[1] Introduction to Liquid Crystals, edited by E. B. Priestley, P. J. Wojtowicz, and P. Sheng (Plenum Press, New York, 1974); The Molecular Physics of Liquid Crystals, edited by G. R. Luckhurst and G. W. Gray (Academic Press, London, 1979).

[2] A. J. Leadbetter and E. K. Norris, Mol. Phys. 38, 669 (1979).

[3] Z. X. Fan and W. Haase, J. Chem. Phys. 95, 6066 (1991).

[4] J. Watanabe and M. Hayashi, Macromolecules 22, 4083 (1989).

[5] Y. Takanishi, A. Ikeda, H. Takezoe, and A. Fukuda, Phys. Rev. E 51, 400 (1995).

[6] N. Kapernaum and F. Giesselmann, Phys. Rev. E 78, 062701 (2008).

[7] G. G. Alexander, S. M. King, R. M. Richardson, and H. Zimmermann, Liq. Cryst. 37, 961 (2010).
[8] M. Cifelli, G. Cinacchi, and L. De Gaetani, J. Chem. Phys. 125, 164912 (2006).

[9] NMR of Ordered Liquids, edited by E. E. Burnell and C. A. de Lange (Kluwer, Dordrecht, 2003).

[10] A. Yethiraj, Z. Sun, R. Y. Dong, and E. E. Burnell, Chem. Phys. Lett. 398, 517 (2004); A. Yethiraj, A. C. J. Weber, R. Y. Dong, and E. E. Burnell, J. Phys. Chem. B 111, 1632 (2007); A. Yethiraj, R. Y. Dong, and E. E. Burnell, Chem. Phys. Lett. 441, 245 (2007); A. C. J. Weber, X. Yang, R. Y. Dong, W. L. Meerts, and E. E. Burnell, ibid. 476, 116 (2009); A. C. J. Weber, X. Yang, R. Y. Dong, and E. E. Burnell, J. Chem. Phys. 132, 034503 (2010).

[11] G. Cinacchi, Chem. Phys. Lett. 416, 238 (2005).

[12] G. Celebre, G. Cinacchi, and G. De Luca, J. Chem. Phys. 129, 094509 (2008). 
[13] M. E. Rose, Elementary Theory of Angular Momentum (John Wiley \& Sons, New York, 1957).

[14] T. L. Hill, Statistical Thermodynamics (Addison-Wesley, Reading, MA, 1960).

[15] G. Celebre, G. De Luca, M. Longeri, and E. Sicilia, J. Chem. Inf. Comput. Sci. 34, 539 (1994).
[16] L. Calucci, M. Geppi, A. Marini, and C. A. Veracini, Phys. Rev. E 82, 041702 (2010).

[17] A. Bondi, J. Phys. Chem. 68, 441 (1964).

[18] I. Nezbeda, Mol. Phys. 103, 59 (2005).

[19] E. H. Pape, Mol. Cryst. Liq. Cryst. 102, 271 (1984). 